\title{
Bone Marrow-Derived Cells Expressing Green Fluorescent Protein under the Control of the Glial Fibrillary Acidic Protein Promoter Do Not Differentiate into Astrocytes
} In Vitro and In Vivo

\author{
Tim Wehner, ${ }^{1 *}$ Matthias Böntert, ${ }^{1 *}$ Ilker Eyüpoglu, ${ }^{2}$ Konstantin Prass, ${ }^{1}$ Marco Prinz, ${ }^{3}$ Francisco Fernández Klett, ${ }^{1}$ \\ Matthias Heinze, ${ }^{1}$ Ingo Bechmann, ${ }^{2}$ Robert Nitsch, ${ }^{2}$ Frank Kirchhoff, ${ }^{4}$ Helmut Kettenmann, ${ }^{5}$ Ulrich Dirnagl, ${ }^{1}$ and \\ Josef Priller ${ }^{1}$ \\ ${ }^{1}$ Department of Neurology and ${ }^{2}$ Institute of Anatomy, Charité, Humboldt-University, 10117 Berlin, Germany, ${ }^{3}$ Institute of Neuropathology, Georg-August- \\ University, 37075 Göttingen, Germany, ${ }^{4}$ Max-Planck-Institute of Experimental Medicine, 37075 Göttingen, Germany, and ${ }^{5}$ Max-Delbrück-Center for \\ Molecular Medicine, 13125 Berlin-Buch, Germany
}

Differentiation of bone marrow (BM) cells into astroglia expressing the glial fibrillary acidic protein (GFAP) has been reported in vitro and after intracerebral or systemic BM transplantation. In contrast, recent data suggest that astrocytic differentiation does not occur from BM-derived cells in vivo. Using transgenic mice that express the enhanced green fluorescent protein (GFP) under the control of the human glial fibrillary acidic protein (GFAP) promoter, we investigated the potential of adult murine BM-derived cells to differentiate into macroglia. In the brains of GFAP-GFP transgenic mice, astrocytes were brightly fluorescent from the expression of GFP. When BM from these animals was transplanted into lethally irradiated wild-type animals, the transgene was detected in the reconstituted hematopoietic system, but no GFP expression was found in the nervous system. In contrast, GFAP-GFP neuroectodermal anlage grafted into adult wild-type striatum gave rise to GFP-expressing astrocytes. Because cerebral ischemia has been suggested to promote the differentiation of BM-derived cells into astrocytes, BM chimeric mice were subjected to focal cerebral ischemia. No GFP-positive cells were found in the ischemic or contralateral hemispheres of these brains. Even after direct injection of GFAP-GFP transgenic BM cells into wild-type striatum, no GFP-expressing astroglia were detected. To test the hypothesis that the in vitro environment might be more permissible for astroglial differentiation, we cultured BM from mice that constitutively express GFP, BM cells expressing GFP from a retroviral vector, and BM from GFAP-GFP transgenic mice on astrocytes and on organotypic hippocampal slices. In all experimental paradigms, BMderived cells were found to differentiate into ramified microglia but not into GFAP-expressing astrocytes.

Key words: astroglia; microglia; bone marrow transplantation; green fluorescent protein; cell culture; cerebral ischemia

\section{Introduction}

Astrocytes are the most numerous glial cells of the CNS and are thought to derive from multipotent and glial-restricted progenitors, including O-2A progenitors (Mehler and Gokhan, 1999). Recently, astrocytes have themselves been suggested to act as neural stem cells, capable of generating macroglia and neurons (Doetsch et al., 1999; Seri et al., 2001; Heins et al., 2002). Moreover, astrocytes from hippocampus appear to stimulate adult neurogenesis (Song et al., 2002). Although macroglia, i.e., astrocytes and oligodendrocytes, have traditionally been considered neuroectodermal of origin, recent experiments using radiation bone marrow (BM) chimeras have suggested that $\sim 0.5 \%$ of CNS astrocytes may derive from the bone marrow (Eglitis and Mezey, 1997; Eglitis et al., 1999). Marrow stromal cells were found to differentiate into astrocytes after intraventricular injection into

\footnotetext{
Received Dec. 4, 2002; revised March 17, 2003; accepted March 26, 2003.

This work was partly supported by the Deutsche Forschungsgemeinschaft-Sonderforschungsbereich 507 and the Hermann and Lilly Schilling Foundation.

Correspondence should be addressed to Dr. Josef Priller, Department of Neurology, Charité, HumboldtUniversity, Schumannstrasse 20/21, 10117 Berlin, Germany. E-mail: josef.priller@charite.de.

Copyright $\odot 2003$ Society for Neuroscience $\quad$ 0270-6474/03/235004-08\$15.00/0
}

neonatal mouse brains and after intravenous administration after cerebral ischemia in rats (Kopen et al., 1999; Chen et al., 2001). Moreover, intravenous and intrastriatal transplantation of genetically marked BM cells into irradiated mice led to the generation of some GFAP-expressing astrocytes (Nakano et al., 2001; Corti et al., 2002a,b). Even more surprisingly, BM-derived cells have recently been shown to differentiate into cells expressing neuronal antigens in vivo (Brazelton et al., 2000; Mezey et al., 2000; Priller et al., 2001a). Yet, among 8000 astrocytes counted by Brazelton et al. (2000), not a single cell was derived from the bone marrow. Neither Nakano et al. (2001) nor Hess et al. (2002) were able to detect donor-derived astrocytes in radiation bone marrow chimeras.

Controversy also exists with regard to the in vitro data on the differentiation of BM stromal cells into astroglia. Although Sanchez-Ramos et al. (2000), Kohyama et al. (2001), and Jiang et al. (2002) found that mouse and human marrow stromal/mesenchymal cells developed into astrocytes in vitro, Woodbury et al. (2000) and Deng et al. (2001) could not detect any generation of astroglia from adult rat and human BM stromal cells.

In this study, we used transgenic mice that express enhanced 
green fluorescent protein (GFP) under the control of the human GFAP promoter (Nolte et al., 2001) to address the issue of astroglial differentiation of BM-derived cells. GFAP is the principal 8-9 nm intermediate filament protein in mature astrocytes of the CNS (Eng et al., 2000). The GFAP promoter has been well characterized (for review, see Brenner, 1994). In our GFAP-GFP transgenic mice, strong expression of the GFP fluorophore was detected in both protoplasmic and fibrous astrocytes. The level of GFP expression varied with age and the brain region examined. Radial glia from the transgenic mice expressed GFP and differentiated into GFAP-positive astrocytes in vitro (M. Götz, unpublished observations). Similar results were obtained in a transgenic mouse line expressing hGFP-S65T under the human GFAP promoter (Malatesta et al., 2000). When transplanted into irradiated wild-type mice or injected into the ischemic striatum of wild-type mice, we did not find GFAP-GFP transgenic BM-derived cells to differentiate into astrocytes. Moreover, these cells did not acquire an astroglial phenotype in vitro. Under the same experimental conditions, BM cells that constitutively express GFP differentiated into ramified microglia.

\section{Materials and Methods}

Animals. Transgenic mice expressing GFP under the control of the human GFAP promoter were generated on FVB/N background (Nolte et al., 2001). Eight- to 12-week-old wild-type FVB/N and FVB/N $\times$ C57BL/6 mice (Charles River, Sulzfeld, Germany) were used as BM recipients in transplantation studies and for intracerebral grafting. BM obtained from adult NMRI mice (Charité breeding facility, Berlin, Germany) and from GFAP-GFP transgenic mice crossed into the C57BL/6 strain was used for retroviral transduction. BM cells were cultured on astrocytes or on hippocampal slices from newborn NMRI mice. Transgenic mice expressing GFP under the control of the $\beta$-actin promoter were generated on C57BL/6 background (Okabe et al., 1997). These mice were generously provided by Dr. Okabe (Osaka University, Japan). All animal procedures were performed under the local guidelines for animal use in experimental laboratory research.

Retroviral transduction of bone marrow cells. BM was harvested from femurs and tibias $48 \mathrm{hr}$ after treatment with $150 \mathrm{mg} / \mathrm{kg} 5$-fluoruracil (Sigma, Deisenhofen, Germany). Transduction of BM cells with a murine stem cell virus-based retroviral vector containing the GFP cDNA was performed as described in Priller et al. (2001b). Briefly, BM cells were cultured in DMEM supplemented with 15\% fetal calf serum (FCS; Biochrom, Berlin, Germany), $50 \mathrm{ng} / \mathrm{ml}$ rat stem cell factor (generously provided by Amgen, Thousand Oaks, CA), $20 \mathrm{ng} / \mathrm{ml}$ interleukin-3, and 50 $\mathrm{ng} / \mathrm{ml}$ interleukin-6 (Promocell, Heidelberg, Germany). After 2 d, BM cells were transferred onto irradiated (13 Gy) viral producer cells and cultured for another $48 \mathrm{hr}$. Nonadherent BM cells were finally rinsed off the producer cell monolayer. Efficiency of gene transfer into clonogenic hematopoietic precursors was assessed in colony assays on methylcellulose supplemented with hematopoietic cytokines (Stem Cell Technologies, Vancouver, British Columbia, Canada).

Bromodeoxyuridine labeling. BM cells were exposed to $3 \mu \mathrm{M}$ bromodeoxyuridine (BrdU; Sigma) during the above described retroviral transduction procedure. BrdU was supplemented daily for 4 consecutive days. BrdU-labeled BM cells were washed several times in PBS before culture on astrocytes.

Generation of GFAP-GFP bone marrow chimeras. BM transplantation was performed as described previously (Priller et al., 2001b). BM cells $\left(5 \times 10^{6}\right)$ were resuspended in PBS/2\% FCS and injected intravenously into lethally irradiated (two doses of 5.5 Gy separated by $3 \mathrm{hr}$ ) recipient mice. In one set of experiments, GFAP-GFP BM cells were transduced with the GFP retroviral vector before transplantation. After transplantation, animals were kept isolated in ventilated filter cages.

Detection of the transgene in blood cell progeny. Six to eight weeks after $\mathrm{BM}$ transplantation, $150 \mu \mathrm{l}$ of peripheral blood was drawn from the tail veins of GFAP-GFP bone marrow chimeric mice. Genomic DNA was isolated using the S.N.A.P. whole-blood DNA isolation kit (Invitrogen, Groningen, The Netherlands). PCR was performed using the following primers for the GFP gene: sense (5'-GCC GCT ACC CCG ACC AC-3'), antisense ( $5^{\prime}$-TTC ACC TTG ATG CCG TTC TTC T- $3^{\prime}$ ). PCR conditions were as follows: $3 \mathrm{~min}$ of $94^{\circ} \mathrm{C}, 40$ cycles of $94^{\circ} \mathrm{C}$ for $1 \mathrm{~min}, 55^{\circ} \mathrm{C}$ for $1 \mathrm{~min}, 72^{\circ} \mathrm{C}$ for $1.5 \mathrm{~min}$, final extension at $72^{\circ} \mathrm{C}$ for $5 \mathrm{~min}$. PCR products were separated on a $1 \%$ agarose gel and visualized using ethidium bromide. After transplantation of GFAP-GFP BM cells transduced with the GFP retroviral vector, peripheral blood was drawn from the chimeric mice and leukocytes were analyzed for GFP expression by FACS (Becton Dickinson, Heidelberg, Germany).

Induction of focal cerebral ischemia. Transient focal cerebral ischemia (Hara et al., 1996) was induced in GFAP-GFP bone marrow chimeric mice 12 weeks after BM transplantation. In a separate set of experiments, chimeras were used that had received GFAP-GFP transgenic BM cells transduced with the GFP retroviral vector. During surgery, general anesthesia was maintained with $1.0 \%$ halothane in $70 \% \mathrm{~N}_{2} \mathrm{O} / 30 \% \mathrm{O}_{2}$. Body temperature was kept at $37-37.5^{\circ} \mathrm{C}$ using a heating pad. The left middle cerebral artery was occluded by insertion of a silicone-coated monofilament (Heraeus Kulzer, Hanau, Germany) via the left internal carotid artery. Onset of ischemia was verified by measuring the regional cerebral blood flow using laser Doppler flowmetry. After $60 \mathrm{~min}$ of ischemia, the monofilament was withdrawn to reperfuse the brain.

Intracerebral grafting procedures. Wild-type mice were subjected to 60 min of focal cerebral ischemia as described above. After $3 \mathrm{hr}, 5 \times 10^{5} \mathrm{BM}$ cells from GFAP-GFP transgenic mice were injected into the left ischemic striatum. Precise positioning of the grafts was verified in control mice injected with yellow-green fluorescent polystyrene microspheres (Molecular Probes, Leiden, The Netherlands). After surgery, animals were kept isolated in ventilated filter cages.

Embryos of GFAP-GFP transgenic mice were obtained at day 12.5 after conception and transferred into modified Hank's medium supplemented with $10 \%$ FCS, $2 \%$ glucose at $4^{\circ} \mathrm{C}$. The neuroectodermal anlage was prepared, homogenized in a volume of $30 \mu \mathrm{l}$, and grafted into the caudoputamen of adult wild-type mice as described previously (Isenmann et al., 1996). Similarly, $5 \times 10^{5}$ BM cells from GFAP-GFP transgenic mice were grafted into the caudoputamen of adult wild-type mice.

Evaluation of donor cell engraftment in the CNS. At given time points, mice were killed by intraperitoneal injection with chloralhydrate, followed by transcardial perfusion with ice-cold PBS for 1-2 min and 4\% paraformaldehyde (PFA) for $15 \mathrm{~min}$. The brains were removed, postfixed in $4 \%$ PFA, and sectioned using a cryotome $(20 \mu \mathrm{m})$ or a vibratome (30 $\mu \mathrm{m})$. Every 10th section was mounted onto glass slides and examined by conventional fluorescence microscopy (Leica, Heidelberg, Germany) or by confocal laser scanning microscopy (Zeiss, Jena, Germany). Adjacent sections were stained with hematoxylin and eosin. Representative vibratome sections were immunostained for GFAP as described below.

Preparation of astroglial cell cultures. Astrocytes were prepared from newborn mice [postnatal day (P) 1-3] according to the method of McCarthy and De Vellis (1980). Briefly, animals were decapitated and the brains were removed. The cortices were mechanically dissociated and washed in PBS. Each cell suspension obtained from two brains was placed in a flask and cultured in DMEM/10\% FCS. After one to two passages, adherent cells were removed with $0.5 \%$ trypsin and plated in 24-well culture plates at a density of 40,000 cells per well in the above medium. Astrocytes were used for coculture experiments when they reached confluency. Astroglial cell cultures were characterized by immunocytochemistry for GFAP as described below.

Preparation of organotypic entorhinal-hippocampal slice cultures. Organotypic entorhinal-hippocampal slice cultures (OEHSCs) were prepared from newborn mice (P9-12) as described previously (Savaskan et al., 2000). Briefly, animals were decapitated, and the brains were removed and immediately transferred into ice-cold MEM. Vibratome sections $(300 \mu \mathrm{m})$ were obtained from the hippocampal region and placed gently onto $0.4 \mu \mathrm{m}$ sterile porous membranes (Millipore, Eschborn, Germany). Brain tissue slices were covered with $1 \mathrm{ml}$ of MEM supplemented with $25 \%$ horse serum and $25 \%$ HBSS. The culture medium was changed 
every other day. After 5-7 d, OEHCS were used for coculture experiments.

Culture of BM cells on astrocytes and OEHCS. BM cells were seeded either onto astrocytic monolayers at a density of 10,000 cells per well or onto OEHSCs at a density of 100,000 cells per slice. No additional factors were added to the culture medium, which was changed every $48 \mathrm{hr}$. After $1,3,6,12$, and $18 \mathrm{~d}$, astrocyte/BM-cocultures were fixed with 4\% PFA. At each time point, cells growing in select wells were enzymatically mobilized and mounted onto gelatin-coated glass slides by cytocentrifugation at $500 \mathrm{rpm}$ for $10 \mathrm{~min}$ (Becton Dickinson). Slice cocultures were fixed with $4 \%$ PFA/0.1\% glutaraldehyde and snap frozen after 3, 5, and $8 \mathrm{~d}$. Sections $(14 \mu \mathrm{m})$ were obtained on a cryostat and mounted onto gelatincoated glass slides. All samples were examined for GFP expression by conventional fluorescence microscopy. GFP-expressing cells were characterized immunohistochemically as described below.

Immunohistochemistry. Immunohistochemistry was performed as described in Priller et al. (2001b). Cultures or sections were incubated overnight with primary antibodies against GFAP (Dako, Carpinteria, $\mathrm{CA}$ ) or Iba1 (generously provided by Dr. Imai, Tokyo, Japan) at dilutions of 1:500 and 1:100, respectively. A biotinylated secondary antibody (Vector, Burlingame, CA) was added at a dilution of 1:200 for $1 \mathrm{hr}$, followed by visualization of the staining using streptavidin-conjugated Texas Red or Cy3 dyes (Vector). For BrdU immunodetection, fixed cultures were incubated in $2 \mathrm{~N} \mathrm{HCl}$ at $37^{\circ} \mathrm{C}$ for $1 \mathrm{hr}$ followed by permeabilization with 0.3\% Triton X-100 (Sigma) for $30 \mathrm{~min}$. Primary antibodies against BrdU (Harlan, Borchen, Germany) and GFP (Clontech, Palo Alto, CA) were added overnight at dilutions of 1:200 and 1:250, respectively. Secondary antibodies conjugated to Texas Red and Alexa 488 (Molecular Probes) were used for visualization of BrdU and GFP immunoreactivities; omission of primary antibodies served as negative control.

\section{Results}

An overview of the different experimental conditions is shown in Figure 1.

\section{GFAP-GFP transgenic mice}

In the brains of mice that have been genetically engineered to express GFP under the control of the human GFAP promoter, brightly fluorescent stellar cells were detected in the gray and white matter (Fig. $2 A$ ). These cells were identified as astrocytes by their endogenous expression of GFAP (Figs. $2 B, C$ ). Moreover, electrophysiological recordings of GFP-positive cells in the brain revealed behavior characteristic of astroglia (Nolte et al., 2001). Note that not all GFAP-immunoreactive cells expressed detectable levels of GFP (Fig. 2C). The percentage of GFP-expressing astrocytes was $50 \%$ in the cortex, $80 \%$ in the striatum, and $100 \%$ in the cerebellum at the time of BM harvesting. After 1 year, $40 \%$ of the striatal astrocytes expressed GFP (F. Kirchhoff, unpublished observations). Consistent with the cell-specific expression of the transgene in astrocytes, no fluorescence from GFP was detected in BM and peripheral blood cells from GFAP-GFP transgenic mice.

\section{Generation of GFAP-GFP bone marrow chimeras}

Transplantation of GFAP-GFP transgenic BM cells into lethally irradiated wild-type mice led to the reconstitution of hematopoiesis with transgenic peripheral blood cell progeny within 6 weeks (Fig. 3). The percentage of chimerism amounted to up to $70 \%$ in mice transplanted with GFAP-GFP BM cells transduced with a GFP retroviral vector (Priller at al., 2001b) $(n=2)$. No abnormalities of blood counts or blood cell morphology were observed after bone marrow transplantation. The brains of the GFAP-GFP BM chimeric mice $(n=8)$ were analyzed for GFP expression 1-3 months after reconstitution. Every 10th cerebral section was

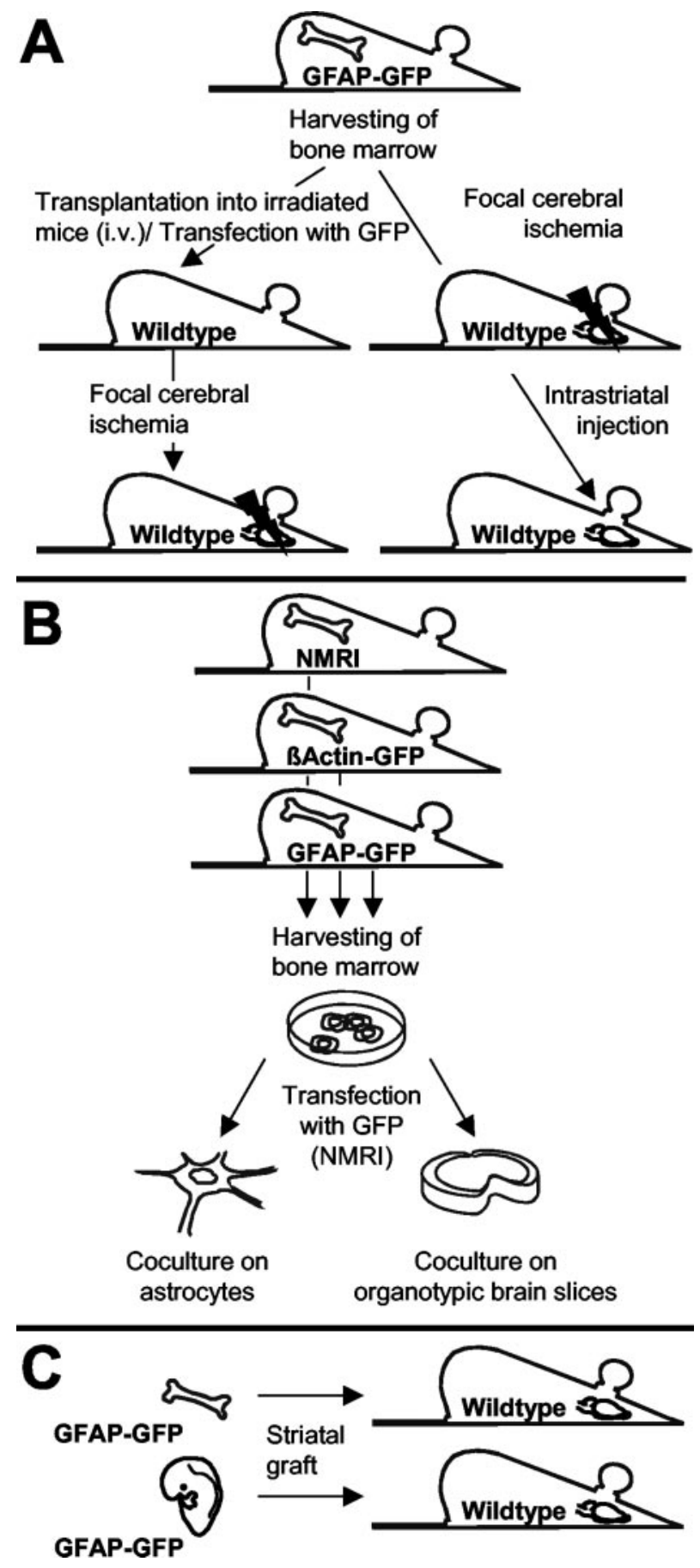

Figure 1. Overview of the different experimental conditions. $A$, In vivo experiments. BM from GFAP-GFP transgenic mice was harvested and transplanted into lethally irradiated adult wild-type mice. The brains of the recipient mice were analyzed for GFP expression before and after focal cerebral ischemia. GFAP-GFP BM cells transduced with the GFP retroviral vector were also transplanted into lethally irradiated adult wild-type mice, and the brains were analyzed for GFP expression after cerebral ischemia. In addition, BM from GFAP-GFP transgenic mice was directly injected into the brains of adult wild-type mice that had been subjected to focal cerebral ischemia. $B$, In vitro experiments. BM from wild-type NMRI mice was harvested and transduced with a retroviral vector containing the GFP marker gene. BM from $\beta$-actin-GFP transgenic mice was also used. BM cells were cultured on astrocytes or on organotypic entorhinal-hippocampal slice cultures. GFP-expressing cells were characterized by immunohistochemistry for GFAP and Iba1. In addition, BM from GFAP-GFP transgenic mice was cultured on astrocytes or on entorhinal-hippocampal slices. The cocultures were analyzed for GFP expression. C, Grafting experiments. Adult BM cells or embryonic neuroectodermal anlage from GFAP-GFP transgenic mice was grafted into the caudoputamen of adult wild-type mice. The brains were analyzed for GFP expression. 

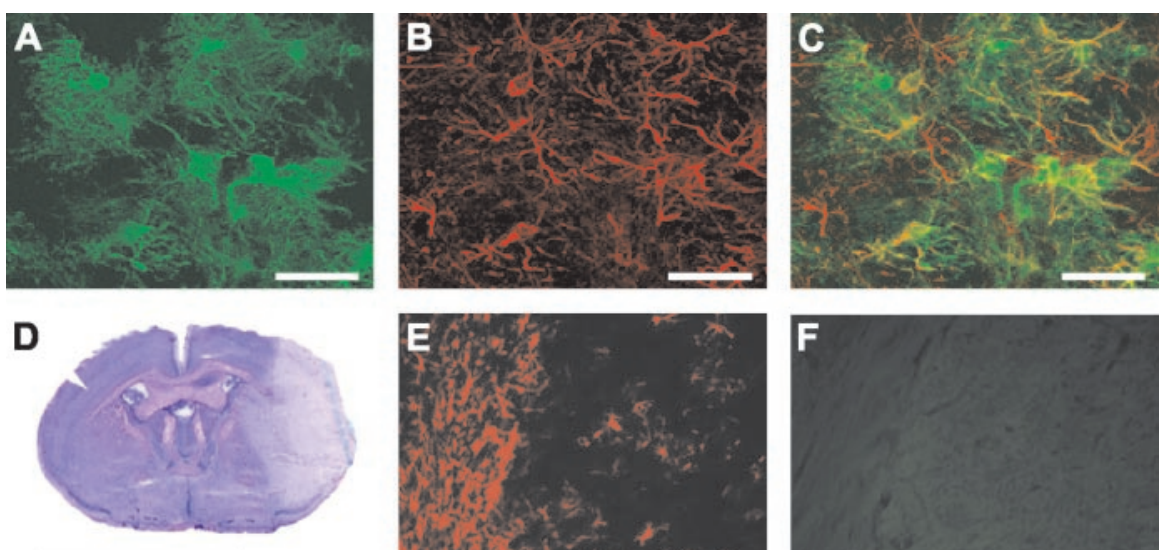

$1 \mathrm{~cm}$
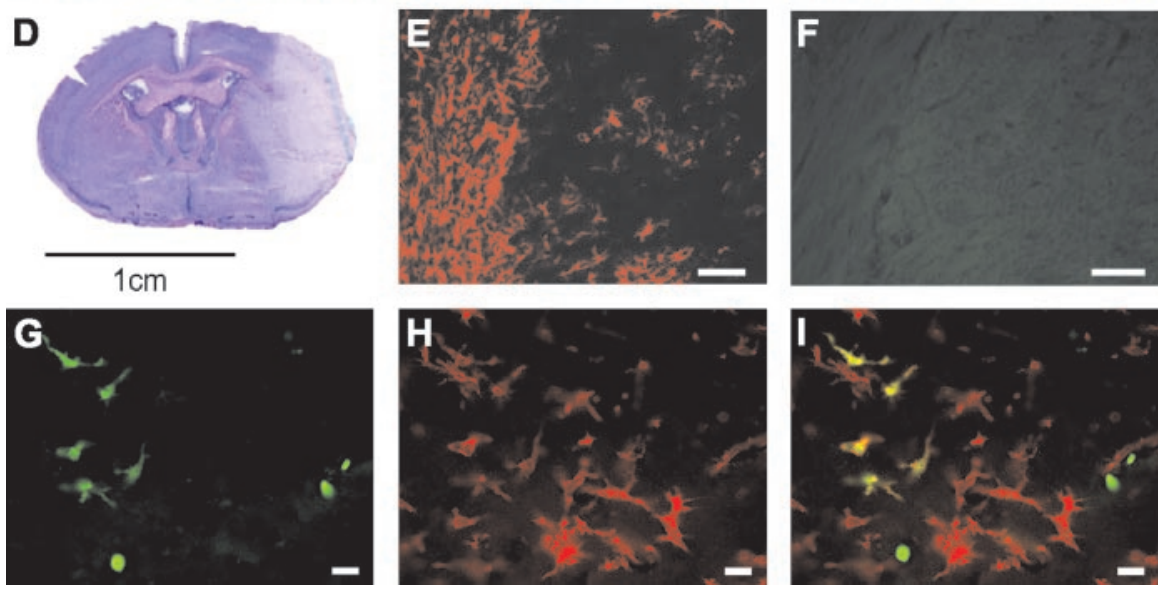

Figure 2. Characterization of transgenic mice that express GFP under the control of the GFAP-promoter (GFAP-GFP mice). $A$, Striatal brain slices were prepared from 3-month-old GFAP-GFP transgenic mice and analyzed by confocal laser scanning microscopy. GFP was expressed in the cytoplasm of protoplasmic astrocytes. $B$, Astrocytes expressed the intermediate filament protein, GFAP, as revealed by immunohistochemistry using Cy3-conjugated secondary antibodies. $C$, Overlay of images $A$ and $B$ demonstrates coexpression but differential subcellular localization of the two proteins. The majority of astrocytes expressed detectable amounts of GFP. The images display projections of $230 \times 130 \times 13 \mu \mathrm{m}$. D-F, Induction of focal cerebral ischemia in GFAP-GFP BM chimeras. Representative hematoxylin and eosin-stained section of a mouse brain $48 \mathrm{hr}$ after MCAO (60 min). The ischemic lesion is visible on the right $(D) . E, F$, Immunohistochemical analysis of GFAP expression. Marked astrogliosis $(E)$ demarcated the border of the ischemic lesion $48 \mathrm{hr}$ after MCAO in a GFAP-GFP BM chimera. No GFP expression could be detected in the same visual field $(F$ ), indicating that GFAP expression did not occur in BM-derived cells. G-I, Five days after MCAO (60 min) in a BM chimera that had received GFAP-GFP transgenic BM cells labeled with GFP using a retroviral vector, donor-derived cells were detectable in the ischemic hemisphere by virtue of their GFP expression ( $G$ ). Immunohistochemistry for Iba1 revealed marked activation of microglia and macrophages in the lesioned tissue $(H)$. Some BM-derived cells have differentiated into microglia, as indicated by their ramified morphology and coexpression of GFP and lba1 (I). Scale bars: $A-F, 50 \mu \mathrm{m} ; G-I, 10 \mu \mathrm{m}$.

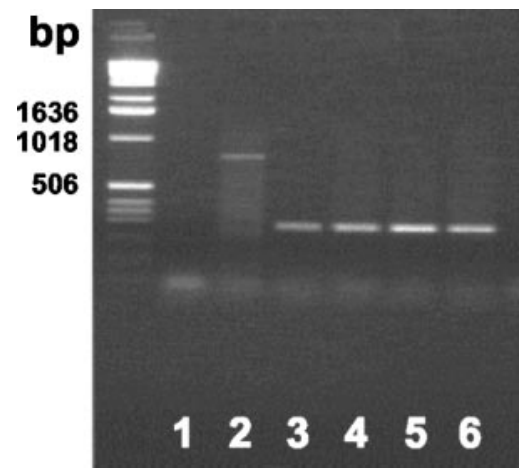

Figure 3. Presence of the transgene in peripheral blood cells from GFAP-GFP bone marrow chimeras. Genomic DNA from peripheral blood leukocytes was analyzed for the presence of the GFP transgene by PCR. PCR products were separated on an agarose gel and stained with ethidium bromide. The migration patterns of molecular weight markers are shown on the left. Lane 1, Negative control $\left(\mathrm{H}_{2} \mathrm{O}\right)$; lanes 2-6, GFAP-GFP BM chimeric mice. Note that the animal from lane 2 has not been successfully reconstituted with GFAP-GFP BM, whereas the chimeras from lanes 3-6 carry transgenic blood cell progeny.

carefully evaluated, but not a single GFP-positive cell was detected. In two chimeras, the entire brains were screened for GFPexpressing cells. No neuropathological changes were observed on hematoxylin and eosin stains of the brain sections.
Induction of transient focal cerebral ischemia in GFAP-GFP bone marrow chimeras

Because cerebral ischemia has been suggested to promote the differentiation of BM-derived cells into astrocytes in vivo (Eglitis et al., 1999), GFAP-GFP BM chimeras were subjected to focal cerebral ischemia by occlusion of the middle cerebral artery for $60 \min (n=6)$. Reperfusion times were $1-5 \mathrm{~d}$. As demonstrated by hematoxylin and eosin staining, all animals developed large left-hemispheric infarcts after middle cerebral artery occlusion (MCAO) (Fig. 2 D). Immunohistochemistry for GFAP revealed marked astrogliosis, demarcating the border of the ischemic core (Fig. 2E). No GFP-expressing cells were detected in ipsilateral (Fig. 2F) and contralateral sections of the ischemic brains when every 10th section was scored. To demonstrate the presence of BMderived cells in the ischemic brain, GFAPGFP transgenic BM cells were transduced with GFP using a murine stem cell virusbased retroviral vector before transplantation into lethally irradiated adult wild-type mice. Four weeks after BM transplantation, the chimeras were subjected to $60 \mathrm{~min}$ of MCAO and 5-14 d of reperfusion $(n=2)$. A large infiltrate of GFP-expressing cells was detected in the ischemic hemisphere (Fig. $2 G$ ), indicating that donor-derived cells engraft and survive in the lesioned brain. Ramified GFP-expressing cells were immunoreactive for Ibal (Figs. $2 \mathrm{H}, \mathrm{I}$ ), suggesting that BM-derived cells differentiated into microglia (Imai et al., 1996).
Intracerebral grafts of GFAP-GFP transgenic cells

On the basis of previous results reported by Kopen et al. (1999) and Nakano et al. (2001), we presumed that blood cells might differentiate more easily into brain cells from intracerebral grafts of bone marrow. Thus, GFAP-GFP BM cells were injected into the ischemic striatum of wild-type mice $3 \mathrm{hr}$ after $\operatorname{MCAO}(n=4)$. The correct positioning of the grafts was confirmed by hematoxylin and eosin staining visualizing the needle tract. No GFPexpressing astrocytes were detected in the striatal grafts up to $7 \mathrm{~d}$ after transplantation. When GFAP-GFP BM cells were grafted into the caudoputamen of wild-type mice $(n=4)$, an astroglial scar was detected around the injection site at 6 weeks after grafting, but none of the cells were expressing GFP (Figs. $4 A-C$ ). In contrast, E12.5 neuroectodermal tissue from GFAP-GFP transgenic mice was GFP negative at the time of grafting into the caudoputamen of wild-type mice $(n=4)$ but gave rise to numerous GFP-expressing astrocytes within 6 weeks (Figs. $4 D-F$ ). Thus, the adult murine brain retains the potential to induce an astroglial fate in transplanted stem/progenitor cells.

\section{Differentiation of bone marrow-derived cells into microglia on astrocyte cultures}

To evaluate the potential of BM cells to differentiate into neuroglia in vitro, we transferred the GFP gene into BM cells using the 
above mentioned retroviral vector. Efficiency of gene transfer into myeloid clonogenic progenitors was assessed to be $>90 \%$. The fluorescence from GFP in BM cells was intense and stable over $>3$ weeks in culture. GFP-expressing BM cells were cultured on astroglial monolayers $(n=4$ with five time points each and four wells per time point). In a parallel study, prelabeling of GFP-expressing BM cells with BrdU was performed to account for BM cells that failed to express GFP ( $n=2$ with three time points each and two to six wells per time point). BM cells were significantly smaller than the underlying astrocytes on day 1 of the coculture. During the first days of coculture, $10-20 \%$ of GFP-expressing cells started to ramify and by day 6 acquired the morphology of "resting" microglia (Fig. 5A). The number and morphology of ramified GFP-positive cells did not change between days 7 and 21 of coculture. No ramification of GFPexpressing cells was observed when BM cells were cultured on polycarbonate dishes in the absence of astrocytes. The ramified GFP-expressing cells were identified as microglia by their immunoreactivity for the Iba1 antigen (Figs. 5C,E), an intracellular epitope expressed by monocytes, macrophages, and microglia (Imai et al., 1996). Not a single GFP-positive cell was found to express GFAP after up to $21 \mathrm{~d}$ of coculture (Figs. $5 B, D, F$ ). To exclude any misleading overlay of the GFP and GFAP signals in astrocyte cultures, cells from select wells were enzymatically dissociated and analyzed for GFP and GFAP expression on a single cell level. Thus, 1500 cells were evaluated, but no GFP-positive cell was found to express GFAP. The number of BrdU-labeled cells in the coculture remained fairly constant between 6 and $18 \mathrm{~d}$ in vitro, and most of the GFP-expressing cells were immunoreactive for BrdU (Figs. $5 G-I)$. The ratio of BrdU- versus GFP-positive cells was $\sim 2: 1$.

When bone marrow from transgenic mice that constitutively express GFP under the control of the $\beta$-actin promoter (Okabe et al., 1997) was cultured on astrocytes, differentiation of BMderived cells into Iba1-positive microglia, but not into GFAPexpressing astrocytes, was observed ( $n=1$ with five time points and four wells per time point). Moreover, when BrdU-labeled BM from transgenic mice that express GFP under the control of the GFAP promoter was cultured on astrocytes, no GFP expression was detected in the cocultures after up to $18 \mathrm{~d}$, and BMderived cells were identified by their immunoreactivity for BrdU ( $n=2$ with four time points and two wells per time point). These findings in two independent transgenic models confirm the above results obtained with BM cells transduced with a retroviral vector.

\section{Differentiation of bone marrow-derived cells into microglia on OEHSCs}

To take advantage of an organotypic microenvironment, GFPtransduced BM cells were seeded on top of entorhinal-hippocampal slice cultures $(n=3$ with three time points each and four to six slices per time point). Duration of coculture experiments was limited to $8 \mathrm{~d}$ because of the progressive astrogliosis of the brain slices. Using fluorescence microscopy of living and fixed slice cultures, GFP-expressing cells were found to migrate from the top into the deeper layers of the tissue. As early as $3 \mathrm{~d}$ after inoculation, ramified GFP-expressing cells were detected in various depths of the slice cultures (Fig. 5J). These cells stained positive for the Ibal antigen, indicating their microglial identity (Figs. $5 K, L$ ). At no time point were GFAP-expressing astrocytes of bone marrow origin detected.

When BM from transgenic mice that constitutively express GFP under the control of the $\beta$-actin promoter was cultured on OEHSCs, differentiation of BM-derived cells into Iba1-positive microglia, but not into GFAP-expressing astrocytes, was observed ( $n=1$ with three time points and four slices per time point). Moreover, when BM from transgenic mice that express GFP under the control of the GFAP promoter was cultured on OEHSCs, no GFP expression was detected in the cocultures after up to $8 \mathrm{~d}$ ( $n=1$ with three time points and four slices per time point). These findings in two independent transgenic models confirm the above results obtained with BM cells transduced with a retroviral vector.

\section{Discussion}

Our results add to the controversy regarding the origin of astrocytes in the adult rodent brain. Brazelton et al. (2000) recently suggested that microglia/macrophages and neuron-like cells but not astrocytes were generated from the bone marrow that reconstituted hematopoiesis in lethally irradiated adult mice. As in our protocol, the green fluorescent protein was used to mark BMderived cells. Similarly, Nakano et al. (2001) failed to detect any donor-derived astrocytes in chimeras transplanted with BM cells expressing GFP under the control of the $\beta$-actin promoter. In contrast, Corti et al. (2002a,b) found some GFAP-expressing astrocytes in $\beta$-actin-GFP bone marrow chimeras. Recently, transplantation of multipotent adult progenitor cells, which can differentiate into all neuroectodermal lineages in vitro and after injection into an early blastocyst, did not result in engraftment of donor-derived astrocytes in the adult brain (Jiang et al., 2002). In contrast, Eglitis and Mezey (1997) reported that $0.5-2 \%$ of the BMderived cells that had engrafted in the CNS after transplantation of male $\mathrm{BM}$ cells into lethally irradiated female WBB6F1/J-Kit ${ }^{\mathrm{w}} / \mathrm{J}-$ 

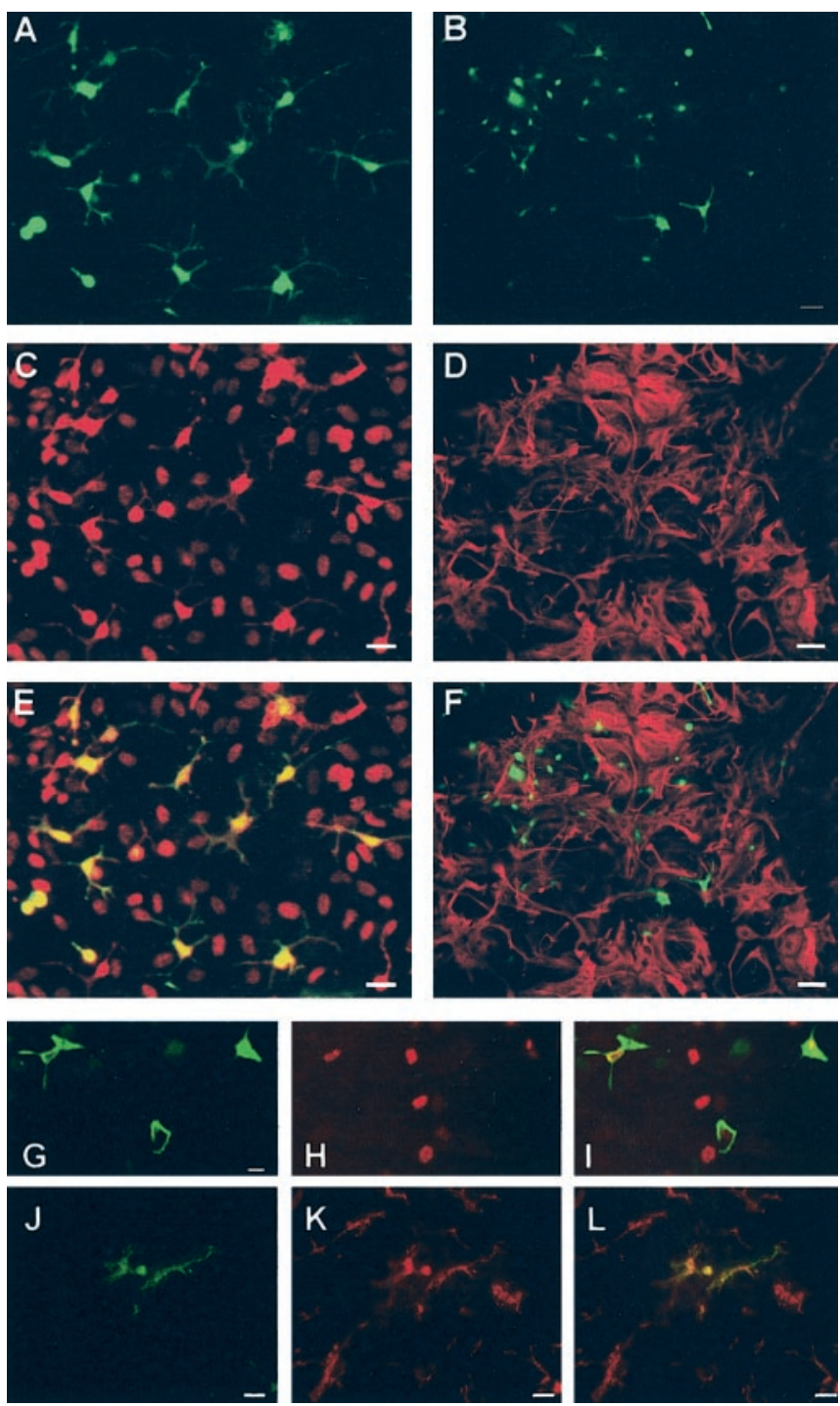

Figure 5. Differentiation of GFP-transduced bone marrow cells into microglia on cultured astrocytes and on OEHSC. A-I, Retroviral transfer of the GFP gene into wild-type BM cells was followed by culture of these cells on astroglial monolayers. After $6 \mathrm{~d}$ of coculture, GFPexpressing BM-derived cells were highly ramified $(A, B)$. Immunohistochemical analysis revealed that all ramified GFP-expressing cells $(A)$ were lba1 positive ( $C$. Images in $A$ and $C$ are superimposed in $E$. No colocalization of the GFP signal $(B)$ with GFAP $(D)$ is observed. Images in $B$ and $D$ are superimposed in $F$. Note that the GFAP immunoreactivity in $D$ results from the astrocytic monolayer on which BM cells were cultured. Six days after culture on astroglia of BrdU-labeled BM cells transduced with the GFP retroviral vector, GFP-expressing cells $(G)$ showed nuclear BrdU immunoreactivity $(H)$. Images in $G$ and $H$ are superimposed in $/$. Note that the degree of BrdU immunoreactivity varied and that GFP gene transfer was incomplete, indicated by the presence of numerous BrdU ${ }^{+} \mathrm{GFP}^{-}$cells. $J-L$, Retroviral transfer of the GFP gene into wild-type BM cells was followed by culture of these cells on OEHSCs. After $8 \mathrm{~d}$ of coculture, many GFP-expressing BM-derived cells were ramified ( $)$. Immunohistochemical analysis revealed that the ramified GFP-expressing cells were Iba1 positive $(K)$. Colocalization is demonstrated in the overlay ( $L$ ). Not all Iba1-expressing cells were GFP positive, because most of the microglia and macrophages in the coculture were derived from the original slice tissue. Scale bars: $A, C, E, 10 \mu \mathrm{m} ; B, D, F, 20 \mu \mathrm{m} ; G, H, I, 5 \mu \mathrm{m} ; J, K, L, 10 \mu \mathrm{m}$.

$\mathrm{Kit}^{\mathrm{W}-\mathrm{v}}$ mice expressed GFAP. It should be noted that the recipient mouse strain used in their study was genetically defective in stem cells. Moreover, characterization of engrafted BM cells required in situ hybridization histochemistry for the Y chromosome combined with immunohistochemistry for GFAP. Using GFP as a reporter driven by the GFAP promoter, we avoided challenging doublelabeling studies. Moreover, we excluded the possibility that antibod- ies may recognize a GFAP-like epitope rather than astrocytic GFAP. On the other hand, the human promoter driving transgene expression in our mice may not replicate exactly the regulatory properties of the endogenous GFAP promoter and appears to be susceptible to gene silencing in vivo. To exclude the possibility that GFP expression was simply below the limit of detection, or that some astrocytes even failed to activate the transgenic promoter, GFAP-GFP transgenic BM cells were transduced with a GFP retroviral vector before transplantation. In accordance with previously published data using GFP-expressing bone marrow (Nakano et al., 2001; Priller et al., 2001b), the donor-derived cells acquired a microglial phenotype in the CNS. Although specific BM stem cell populations giving rise to astroglia may have been selected against in vitro in these experiments, it should be noted that the stem cells generating astroglia in the study of Eglitis and Mezey (1997) had also undergone retroviral transduction before transplantation.

Cramer and Chopp (2000) recently suggested that recovery from cerebral ischemia recapitulates ontogeny. The microenvironment of the ischemic brain therefore may promote the differentiation of BM-derived cells into astrocytes. As a matter of fact, Eglitis et al. (1999) found that $4-5 \%$ of the Y chromosomepositive, BM-derived cells that had engrafted in the ischemic brains of chimeric hypertensive rats within $48 \mathrm{hr}$ were also GFAP positive. Chen et al. (2001) reported that 5\% of bromodeoxyuridine-labeled BM stromal cells infused into ischemic rats expressed GFAP. In the present study, no GFAP-GFP BMderived cells were found to differentiate into astrocytes in the ischemic mouse brain. Hess et al. (2002) also failed to detect donor-derived astrocytes in GFP bone marrow chimeras after MCAO. We confirmed engraftment of transgenic BM cells in the lesioned brain by retroviral GFP labeling of the bone marrow before transplantation. The engrafted GFP-expressing cells were identified as microglia/macrophages. It should be noted, however, that the duration of MCAO in our study was substantially shorter than in the previous experiments of Eglitis et al. (1999) and Chen et al. (2001), and we used a different species.

Immunodepleted marrow stromal cells labeled with bromodeoxyuridine after $7 \mathrm{~d}$ in culture and injected into the ventricle of newborn (P3) mice were reported to migrate throughout the forebrain and cerebellum and to differentiate into GFAPexpressing astrocytes (Kopen et al., 1999). GFP-expressing BM cells injected into the striatum of an irradiated adult mouse were found to differentiate into astrocytes, oligodendrocytes, and microglia, but none turned into neurons (Nakano et al., 2001). Zhao et al. (2002) recently detected macroglia and neuronal phenotypes derived from human BM stem cells grafted into the ischemic brains of spontaneously hypertensive rats. However, Hofstetter et al. (2002) found that marrow stromal cells transplanted into the injured spinal cord promoted recovery but failed to differentiate into GFAP-expressing astrocytes. When we grafted GFAP-GFP transgenic BM cells into the striatum of adult wildtype mice, no differentiation of BM cells into GFP-expressing astrocytes was observed. In contrast, embryonic neuroectodermal tissue from GFAP-GFP transgenic mice gave rise to GFPpositive astrocytes when grafted into adult wild-type mice. This is an important control because it demonstrates that the transgene can be expressed in cells transplanted into the adult brain. Moreover, the microenvironment of the adult brain apparently allows for astroglial differentiation of grafted cells. It is conceivable that slight variations in protocol, such as differences in age and condition of the recipient animals, injection site, and selection of specific BM stem cell populations before transplantation may account for the discrepancies observed between this and previous 
studies. In fact, our approach is different from the above mentioned studies in that BM cells were injected directly into the brain without in vitro treatment, which may have induced predifferentiation.

The GFP molecule has been expressed in a wide variety of cultured cells without deleterious effects (Cheng et al., 1996). In a transgenic model, GFP was placed under the control of an oligodendroglial promoter, and the differentiation of glial cells was not affected (Fuss et al., 2000). The GFAP-GFP transgenic mice used in this study express GFP from birth to adulthood (Nolte et al., 2001; present study). Neurografts from the transgenic mice give rise to $\mathrm{GFP}^{+} \mathrm{GFAP}^{+}$astrocytes (present study). Neither astroglial morphology nor function is altered in the transgenic mice. Mice transplanted with GFAP-GFP BM cells engrafted with normal kinetics, as indicated by the presence of the transgene in peripheral blood leukocytes 8 weeks after transplantation. The fact that GFP expression does not interfere with the differentiation of BM-derived cells into glia is underscored by the generation of Iba1-positive microglia from GFP-marked bone marrow. GFP-expressing BM cells cultured on astrocytes or on hippocampal slice cultures were found to adopt the morphology of ramified microglia within 1 week of coculture. These results extend previous findings suggesting that blood monocytes and spleen macrophages ramify and show the properties of microglia when cultured on astrocytes for 8-14 d (Sievers et al., 1994). As in our study, the absence of astroglia prevented the ramification of blood-borne cells, suggesting that diffusible factors do not suffice for microglial differentiation of BM-derived cells.

No GFAP-expressing cells of bone marrow origin were detected when either GFP-transduced BM cells, BM cells that constitutively express GFP, or GFAP-GFP transgenic BM cells were cultured on astrocytes or on hippocampal slice cultures. These results are in line with the observations of Woodbury et al. (2000) and Deng et al. (2001), who reported that adult rat and human bone marrow stromal cells differentiate into neurons but not into GFAP-expressing astrocytes in vitro. In contrast, Sanchez-Ramos et al. (2000), Kohyama et al. (2001), and Jiang et al. (2002) recently suggested that adult bone marrow cells have the capacity to differentiate into macroglia in vitro. Specific culture conditions and treatment of stromal cells with growth factors and a demethylating agent or neurogenic factor, such as Noggin, may have been instrumental in generating macroglia from mesenchymal stem cells.

In conclusion, these data suggest that the generation of CNS astroglia from bone marrow in the adult mouse may not be as robust as reported previously. Additional experiments are required to determine the conditions under which bone marrow stem cells may switch lineage and give rise to GFAP-expressing astrocytes.

\section{References}

Brazelton TR, Rossi FMV, Keshet GI, Blau HM (2000) From marrow to brain: expression of neuronal phenotypes in adult mice. Science 290:1775-1779.

Brenner M (1994) Structure and transcriptional regulation of the GFAP gene. Brain Pathol 4:245-257.

Chen J, Li Y, Wang L, Zhang Z, Lu D, Lu M, Chopp M (2001) Therapeutic benefit of intravenous administration of bone marrow stromal cells after cerebral ischemia in rats. Stroke 32:1005-1011.

Cheng L, Fu J, Tsukamoto A, Hawley RG (1996) Use of green fluorescent protein variants to monitor gene transfer and expression in mammalian cells. Nat Biotech 14:606-609.

Corti S, Locatelli F, Strazzer S, Salani S, Del Bo R, Soligo D, Bossolasco P, Bresolin N, Scarlato G, Comi GP (2002a) Modulated generation of neuronal cells from bone marrow by expansion and mobilization of circulating stem cells with in vivo cytokine treatment. Exp Neurol 177:443-452.
Corti S, Locatelli F, Donadoni C, Strazzer S, Salani S, Del Bo R, Caccialanza M, Bresolin N, Scarlato G, Comi GP (2002b) Neuroectodermal and microglial differentiation of bone marrow cells in the mouse spinal cord and sensory ganglia. J Neurosci Res 70:721-733.

Cramer SC, Chopp M (2000) Recovery recapitulates ontogeny. Trends Neurosci 23:265-271.

Deng W, Obrocka M, Fischer I, Prockop DJ (2001) In vitro differentiation of human marrow stromal cells into early progenitors of neural cells by conditions that increase intracellular cyclic AMP. Biochem Biophys Res Commun 282:148-152.

Doetsch F, Caillé I, Lim DA, García-Verdugo JM, Alvarez-Buylla A (1999) Subventricular zone astrocytes are neural stem cells in the adult mammalian brain. Cell 97:703-716.

Eglitis MA, Mezey É (1997) Hematopoietic cells differentiate into both microglia and macroglia in the brains of adult mice. Proc Natl Acad Sci USA 94:4080-4085.

Eglitis MA, DawsonD, ParkK-W, Mouradian MM (1999) Targeting of marrowderived astrocytes to the ischemic brain. NeuroReport 10:1289-1292.

Eng LF, Ghirnikar RS, Lee YL (2000) Glial fibrillary acidic protein: GFAPthirty-one years (1969-2000). Neurochem Res 25:1439-1451.

Fuss B, Mallon B, Phan T, Ohlemeyer C, Kirchhoff F, Nishiyama A, Macklin WB (2000) Purification and analysis of in vivo differentiated oligodendrocytes expressing the green fluorescent protein. Dev Biol 187:259-274.

Hara H, Huang PL, Panahian N, Fishman MC, Moskowitz MA (1996) Reduced brain edema and infarction in mice lacking the neuronal isoform of nitric oxide synthase after transient MCA occlusion. J Cereb Blood Flow Metab 16:605-611.

Heins N, Malatesta P, Cecconi F, Nakafuku M, Tucker KL, Hack MA, Chapouton P, Barde Y-A, Götz M (2002) Glial cells generate neurons: the role of the transcription factor Pax6. Nat Neurosci 5:308-315.

Hess DC, Hill WD, Martin-Studdard A, Carroll J, Brailer J, Carothers J (2002) Bone marrow as a source of endothelial cells and NeuNexpressing cells after stroke. Stroke 33:1362-1368.

Hofstetter CP, Schwarz EJ, Hess D, Widenfalk J, El Manira A, Prockop DJ, Olson L (2002) Marrow stromal cells form guiding strands in the injured spinal cord and promote recovery. Proc Natl Acad Sci USA 99:2199-2204.

Imai Y, Ibata I, Ito D, Ohsawa K, Kohsaka S (1996) A novel gene ibal in the major histocompatibility complex class III region encoding an EF hand protein expressed in a monocytic lineage. Biochem Biophys Res Commun 224:855-862.

Isenmann S, Brandner S, Aguzzi A (1996) Neuroectodermal grafting: a new tool for the study of neurodegenerative diseases. Histol Histopathol 11:1063-1073.

Jiang Y, Jahagirdar BN, Reinhardt RL, Schwartz RE, Keene CD, OrtizGonzalez XR, Reyes M, Lenvik T, Lund T, Blackstad M, Du J, Aldrich S, Lisberg A, Low WC, Largaespada DA, Verfaillie CM (2002) Pluripotency of mesenchymal stem cells derived from adult marrow. Nature 418:41-49.

Kohyama J, Abe H, Shimazaki T, Koizumi A, Nakashima K, Gojo S, Taga T, Okano H, Hata J-I, Umezawa A (2001) Brain from bone: efficient "metadifferentiation" of marrow stroma-derived mature osteoblasts to neurons with Noggin or a demethylating agent. Differentiation 68:235-244.

Kopen GC, Prockop DJ, Phinney DG (1999) Marrow stromal cells migrate throughout forebrain and cerebellum, and they differentiate into astrocytes after injection into neonatal mouse brains. Proc Natl Acad Sci USA 96:10711-10716.

Malatesta P, Hartfuss E, Götz M (2000) Isolation of radial glial cells by fluorescent-activated cell sorting reveals a neuronal lineage. Development 127:5253-5263.

McCarthy KD, De Vellis J (1980) Preparation of separate astroglial and oligoglial cell cultures from rat cerebral tissue. J Cell Biol 85:890-902.

Mehler MF, Gokhan S (1999) Postnatal cerebral cortical multipotent progenitors: regulatory mechanisms and potential role in the development of novel neural regenerative strategies. Brain Pathol 9:515-526.

Mezey É, Chandross KJ, Harta G, Maki RA, McKercher SR (2000) Turning blood into brain: cells bearing neuronal antigens generated in vivo from bone marrow. Science 290:1779-1782.

Nakano K, Migita M, Mochizuki H, Shimada T (2001) Differentiation of transplanted bone marrow cells in the adult mouse brain. Transplantation 71:1735-1740.

Nolte C, Matyash M, Pivneva T, Schipke CG, Ohlemeyer C, Hanisch U-K, Kichhoff F, Kettenmann H (2001) GFAP promoter-controlled EGFP- 
expressing transgenic mice: a tool to visualize astrocytes and astrogliosis in living brain tissue. Glia 33:72-86.

Okabe M, Ikawa M, Kominami K, Nakanishi T, Nishimune Y (1997) Green mice as a source of ubiquitous green cells. FEBS Lett 407:313-319.

Priller J, Persons DA, Klett FF, Kempermann G, Kreutzberg GW, Dirnagl U (2001a) Neogenesis of cerebellar Purkinje neurons from gene-marked bone marrow cells in vivo. J Cell Biol 155:733-738.

Priller J, Flügel A, Wehner T, Boentert M, Haas CA, Prinz M, Fernández-Klett F, Prass K, Bechmann I, de Boer BA, Frotscher M, Kreutzberg GW, Persons DA, Dirnagl U (2001b) Targeting of gene-modified hematopoietic cells to the central nervous system: use of the green fluorescent protein uncovers microglial engraftment. Nat Med 7:1356-1361.

Sanchez-Ramos J, Song S, Cardozo-Pelaez F, Hazzi C, Stedeford T, Willing A, Freeman TB, Saporta S, Janssen W, Patel N, Cooper DR, Sanberg PR (2000) Adult bone marrow stromal cells differentiate into neural cells in vitro. Exp Neurol 164:247-256.

Savaskan NE, Eyüpoglu IY, Bräuer AU, Plaschke M, Ninnemann O, Nitsch R,
Skutella T (2000) Entorhinal cortex lesion studied with the novel dye Fluoro-Jade. Brain Res 864:44-51.

Seri B, García-Verdugo JM, Mc Ewen BS, Alvarez-Buylla A (2001) Astrocytes give rise to new neurons in the adult mammalian hippocampus. J Neurosci 21:7153-7160.

Sievers J, Schmidtmayer J, Parwaresch R (1994) Blood monocytes and spleen macrophages differentiate into microglia-like cells when cultured on astrocytes. Ann Anat 176:45-51.

Song H, Stevens CF, Gage FH (2002) Astroglia induce neurogenesis from adult neural stem cells. Nature 417:39-44

Woodbury D, Schwarz EJ, Prockop DJ, Black IB (2000) Adult rat and human bone marrow stromal cells differentiate into neurons. J Neurosci Res 61:364-370.

Zhao L-R, Duan W-M, Reyes M, Keene CD, Verfaillie CM, Low WC (2002) Human bone marrow stem cells exhibit neural phenotypes and ameliorate deficits after grafting into ischemic brain of rats. Exp Neurol 174:11-20. 